\title{
OPTIMAL CONTROL PROBLEMS FOR PARABOLIC HEMIVARIATIONAL INEQUALITIES WITH BOUNDARY CONDITIONS
}

\author{
Jin-Mun Jeong, Eun-Young Ju, And Hyun-Min Kim
}

\begin{abstract}
In this paper, we study optimal control problems for parabolic hemivariational inequalities of dynamic elasticity and investigate the continuity of the solution mapping from the given initial value and control data to trajectories. We show the existence of an optimal control which minimizes the quadratic cost function and establish the necessary conditions of optimality of an optimal control for various observation cases.
\end{abstract}

\section{Introduction}

Let $Y$ be a Hilbert space and let $U_{a d} \subset L^{2}(0, T ; Y)(T>0)$ be an admissible control set.

In this paper we study the optimal control problems finding a control $\hat{u} \in U_{a d}$ for a given cost function $J$ governed by a parabolic hemivariational inequality of the form such that

$(1.1)$

$$
\left\{\begin{array}{l}
\inf _{u \in U_{a d}} J(u)=J(\hat{u}) \text { satisfing } \\
\frac{\partial}{\partial t} x(y, t)+\Delta x(y, t)-\operatorname{div} C[\varepsilon(x(y, t))]+\Xi(y, t)=B u(t)+f(t) \text { in } \Omega \times(0, \infty), \\
x(y, t)=0 \text { on } \Gamma_{1} \times(0, \infty), \\
C[\varepsilon(x(y, t))] \nu=-(\beta \cdot \nu) x(y, t) \text { on } \Gamma_{0} \times(0, \infty), \\
\Xi(y, t) \in \varphi(x(y, t)) \text { a.e. }(y, t) \in \Omega \times(0, \infty), \\
x(y, 0)=x_{0}(y) \text { in } \Omega,
\end{array}\right.
$$

where $\Omega$ is a bounded domain in $\mathbb{R}^{N}$ with sufficiently smooth boundary $\Gamma$ and $f$ is a forcing function. Let $x^{0} \in \mathbb{R}^{N}, \beta(x)=x-x^{0}, R=\max _{x \in \bar{\Omega}}\left|x-x^{0}\right|$. The

Received August 25, 2014.

2010 Mathematics Subject Classification. Primary 49K205; Secondary 93C20.

Key words and phrases. hemivariational inequality, existence of solution, boundary stabilization, decay rates.

This research was supported by Basic Science Research Program through the National Research Foundation of Korea(NRF) funded by the Ministry of Education(NRF2014R1A6A3A01009038). 
boundary $\Gamma$ is composed of two pieces $\Gamma_{0}$ and $\Gamma_{1}$, which are nonempty sets and defined by

$$
\Gamma_{0}:=\{x \in \Gamma: \beta(x) \cdot \nu \geq \alpha>0\} \text { and } \Gamma_{1}:=\{x \in \Gamma: \beta(x) \cdot \nu \leq 0\},
$$

where $\nu$ is the unit outward normal vector to $\Gamma$. Here $\dot{u}=\frac{\partial u}{\partial t}, u=\left(u_{1}, \ldots, u_{N}\right)^{T}$ is the displacement, $\varepsilon(u)=\frac{1}{2}\left\{\nabla u+(\nabla u)^{T}\right\}=\frac{1}{2}\left(\frac{\partial u_{i}}{\partial x_{j}}+\frac{\partial u_{j}}{\partial x_{i}}\right)$ is the strain tensor, $\varphi(u)=\left(\varphi_{1}\left(u_{1}\right), \ldots, \varphi_{N}\left(u_{N}\right)\right)^{T}, \varphi_{i}$ is a multi-valued mapping by filling in jumps of a locally bounded function $b_{i}, i=1, \ldots, N$. A continuous map $C$ from the space $S$ of $N \times N$ symmetric matrices into itself is defined by

$$
C[\varepsilon]=a(\operatorname{tr} \varepsilon) I+b \varepsilon \text { for } \varepsilon \in S,
$$

where $I$ is the identity of $S, \operatorname{tr} \varepsilon$ denotes the trace of $\varepsilon$ and $a>0, b>0$. For example, in the case $N=2, C[\varepsilon]=\frac{E}{d\left(1-\mu^{2}\right)}[\mu(\operatorname{tr} \varepsilon) I+(1-\mu) \varepsilon]$, where $E>0$ is Young's modulus, $0<\mu<1 / 2$ is Poisson's ratio and $d$ is the density of the plate.

Let $A$ be a continuous linear operator satisfying Gårding's inequality. Namely, we formulated the problem (1.1) as

$$
\dot{x}+A x-\operatorname{div} C[\varepsilon(x)]+\Xi=B u+f \text { in } \Omega \times(0, \infty) .
$$

The background of these variational problems are physics, especially in solid mechanics, where nonconvex and multi-valued constitutive laws lead to differential inclusions. We refer to $[19,20]$ to see the applications of differential inclusions.

The existence of global weak solutions for a class of hemivariational inequalities has been studied by many authors, for example, parabolic type problems in $[14,15,19,20]$, and hyperbolic types in [16, 22, 23]. Rauch [17] and Miettinen and Panagiotopoulos $[14,15]$ proved the existence of weak solutions for elliptic one. Quite recently, Park et al. [21] proved the regularity for solutions of parabolic hemivariational inequalities of dynamic elasticity in the strong sense and investigate the continuity of the solution mapping from initial data and forcing term to trajectories. For the optimal control problem of systems governed by variational inequalities, see $[4,5,9,10]$. We refer to $[11,18]$ to see the applications of nonlinear variational inequalities. First order necessary conditions for state constraint optimal control problems governed by semilinear elliptic problems have been obtained by Bonnans and Tida [6] using methods of convex analysis (see also [12]).

In this paper, based on the regularity for solutions of the equation (1.1), we study optimal control problems for parabolic hemivariational inequalities of dynamic elasticity in the strong sense and investigate the Gâteaux differential of the solution mapping from the given initial value and control data to trajectories due to the variational approach of Lions [12]. We show existence of optimal controls which minimizes the quadratic cost function and establish the necessary conditions of an optimal control for various observation cases. 
Since we are considering nonlinear hemivariational inequality, it is not easy to obtain the optimality conditions of optimal controls. To avoid this difficulty we introduce the smoothing system corresponding to (1.1) by taking the regularization of the nonlinear term $\Xi(\cdot, \cdot)$ (see (HIE-1) in Section 3).

The paper is organized as follows. In Section 2 we study the regularity and a variational of constant formula for solutions of parabolic hemivariational inequality. The existence and regularity for the nondegenerate nonlinear systems has been developed as seen in Theorem 4.1 of [1] or Theorem 2.6 of [3], and the references therein. Thereafter, in Section 3, we prove the existence and the uniqueness of optimal control for the problem (1.1). In Section 4 we show the optimal control which minimizes the quadratic cost function and establish the necessary conditions of optimality of an optimal control for various observation cases. Here we employ the method of transposition due to Lions and Magenes [13], which is an important and challenging problem to extend the theory to practical nonlinear partial differential equations.

\section{Variational inequalities}

Let $V$ and $H$ be complex Hilbert spaces forming Gelfand triple $V \subset H \subset V^{*}$ with pivot space $H$. For the sake of simplicity, we may consider

$$
\|u\|_{*} \leq|u| \leq\|u\|, \quad u \in V
$$

where $\|\cdot\|_{*}$ is the norm of the element of $V^{*}$. If an operator $A$ is bounded linear from $V$ to $V^{*}$ and generates an analytic semigroup, then it is easily seen that

$$
H=\left\{x \in V^{*}: \int_{0}^{T}\left\|A e^{t A} x\right\|_{*}^{2} d t<\infty\right\}
$$

for the time $T>0$. Therefore, in terms of the intermediate theory we can see that

$$
\left(V, V^{*}\right)_{1 / 2,2}=H
$$

We also assume that there exists a constant $C_{1}$ such that

$$
\|u\| \leq C_{1}\|u\|_{D(A)}^{1 / 2}|u|^{1 / 2}
$$

for every $u \in D\left(A_{0}\right)$, where

$$
\|u\|_{D(A)}=\left(|A u|^{2}+|u|^{2}\right)^{1 / 2}
$$

is the graph norm of $D(A)$. Thus, in what follows we will write $V=(D(A)$, $H)_{1 / 2,2}$ as a matter of convenience. Let $a(\cdot, \cdot)$ be a bounded sesquilinear form defined in $V \times V$ and satisfying Gårding's inequality

$$
\operatorname{Re} a(u, u) \geq c_{0}\|u\|^{2}, \quad c_{0}>0 .
$$

Let $A$ be the operator associated with the sesquilinear form $a(\cdot, \cdot)$ :

$$
(A u, v)=a(u, v), \quad u, v \in V .
$$


Then $A$ is a bounded linear operator from $V$ to $V^{*}$, and $A$ generates an analytic semigroup in both of $H$ and $V^{*}$. It is also known that if $a(\cdot, \cdot)$ is a symmetric quadratic form satisfying (2.2) then $A$ is positive definite and self-adjoint and $D\left(A^{1 / 2}\right)=V$.

Let $\phi: V \rightarrow(-\infty,+\infty]$ be a lower semicontinuous, proper convex function. Then the subdifferential operator $\partial \phi: V \rightarrow V^{*}$ of $\phi$ is defined by

$$
\partial \phi(x)=\left\{x^{*} \in V^{*}: \phi(x) \leq \phi(y)+\left(x^{*}, x-y\right), y \in V\right\} .
$$

First, let us concern with the following perturbation of subdifferential operator;

$$
\left\{\begin{array}{l}
x^{\prime}(t)+A x(t)+\partial \phi(x(t)) \ni B u(t), \quad 0<t \leq T, \\
x(0)=x_{0}
\end{array}\right.
$$

For every $\epsilon>0$, define

$$
\phi_{\epsilon}(x)=\inf \left\{\left\|x-J_{\epsilon} x\right\|_{*}^{2} / 2 \epsilon+\phi\left(J_{\epsilon} x\right): x \in V\right\},
$$

where $J_{\epsilon}=(I+\epsilon \partial \phi)^{-1}$. If $B=\partial \phi$, then the function $\partial \phi_{\epsilon}$ is Fréchet differentiable on $V$ and its Frećhet differential $\partial \phi_{\epsilon}=B_{\epsilon}$ is Lipschitz continuous on $H$ with Lipschitz constant $\epsilon^{-1}$ where $B_{\epsilon}=\epsilon^{-1}\left(I-(I+\epsilon B)^{-1}\right)$ as is seen in Corollary 2.2 in Chapter II of [3]. It is also well known results that $\lim _{\epsilon \rightarrow 0} \phi_{\epsilon}=\phi$ and $\lim _{\epsilon \rightarrow 0} \partial \phi_{\epsilon}(x)=(\partial \phi)^{0}(x)$ for every $x \in D(\partial \phi)$ where $(\partial \phi)^{0}: V \rightarrow V^{*}$ is the minimum element of $\partial \phi$. Now, we introduce the smoothing system corresponding to (NE) as follows.

$$
\left\{\begin{array}{l}
x^{\prime}(t)+A x(t)+\partial \phi_{\epsilon}(x(t))=B u(t), \quad 0<t \leq T, \\
x(0)=x_{0} .
\end{array}\right.
$$

Using the regularity for the abstract linear parabolic equation we have the following result on the equation (NE).

Proposition 2.1. 1) Let $u \in L^{2}(0, T ; U)$ and $x_{0} \in V$ satisfying that $\phi\left(x_{0}\right)<$ $\infty$. Then the equation (NE) has a unique solution

$$
x \in L^{2}(0, T ; V) \cap W^{1,2}\left(0, T: V^{*}\right) \subset C([0, T] ; H),
$$

which satisfies

$$
x^{\prime}(t)=B u(t)-A x(t)-\partial \phi^{0}(x(t))
$$

and

$$
\|x\|_{L^{2} \cap W^{1,2}} \leq C_{2}\left(1+\left\|x_{0}\right\|+\|u\|_{L^{2}(0, T ; U)}\right),
$$

where $C_{2}$ is a constant and $L^{2} \cap W^{1,2}=L^{2}(0, T ; V) \cap W^{1,2}\left(0, T ; V^{*}\right)$.

2) Let $a(\cdot, \cdot)$ be a symmetric quadratic form satisfying $(2.2)$ and the following hypothesis hold:

(A) There exists $h \in H$ such that for every $\epsilon>0$ and any $\in D(\phi)$

$$
J_{\epsilon}(y+\epsilon h) \in D(\phi) \text { and } \phi\left(J_{\epsilon}(y+\epsilon h)\right) \leq \phi(y) .
$$


Then for $k \in L^{2}(0, T ; H)$ and $x_{0} \in \overline{D(\phi)} \cap V$ the equation (NE) has a unique solution

$$
x \in L^{2}(0, T ; D(A)) \cap W^{1,2}(0, T ; H) \cap C([0, T] ; H),
$$

which satisfies

$$
\|x\|_{L^{2} \cap W^{1,2} \cap C} \leq C_{2}\left(1+\left\|x_{0}\right\|+\|u\|_{L^{2}(0, T ; U)}\right) .
$$

If $V$ is compactly embedded in $H$, the following embedding

$$
\left.L^{2}(0, T ; V) \cap W^{1,2}\left(0, T ; V^{*}\right) \subset L^{2}(0, T ; H)\right)
$$

is compact in view of Theorem 2 of Aubin [1]. Hence, the mapping $u \mapsto x$ is compact from $L^{2}(0, T ; U)$ to $L^{2}(0, T ; H)$.

Now we give the assumption on the nonlinear terms as follows. that

(F) Let $f$ be a nonlinear single valued mapping from $V$ into $H$. We assume

$$
\left|f\left(t, x_{1}\right)-f\left(t, x_{2}\right)\right| \leq L\left\|x_{1}-x_{2}\right\|
$$

for very $x_{1}, x_{2} \in V$.

Now, we introduce smoothing system corresponding to (NCE) as follows.

$$
\left\{\begin{array}{l}
\frac{d x(t)}{d t}+A x(t)+\partial \phi_{\epsilon}(x(t))=f(t, x(t))+B u(t), \quad 0<t \leq T, \\
x(0)=x_{0} .
\end{array}\right.
$$

Since $-A$ generates a semigroup $S(t)$ on $H$, the mild solution of (SCE) can be represented by

$$
x_{\epsilon}(t)=S(t) x_{0}+\int_{0}^{t} S(t-s)\left\{f\left(s, x_{\epsilon}(s)\right)+B u(s)-\partial \phi_{\epsilon}\left(x_{\epsilon}(s)\right)\right\} d s .
$$

We establish the following result on the solvability of (NCE) as is seen in Theorem 3.7 of [21].

Proposition 2.2. 1) Let $x_{0} \in V$ satisfying that $\phi\left(x_{0}\right)<\infty, u \in L^{2}(0, T ; U)$ and the assumption $(\mathrm{F})$ be satisfied. Then the equation (NCE) has a unique solution

$$
x \in L^{2}(0, T ; V) \cap W^{1,2}(0, T ; V) \cap C([0, T] ; H),
$$

which satisfies

$$
x^{\prime}(t)=f(t, x(t))+B u(t)-A x(t)-\partial \phi^{0}(x(t))
$$

and there exists a constant $C_{3}$ depending on $T$ such that

$$
\|x\|_{L^{2} \cap W^{1,2}} \leq C_{3}\left(1+\left\|x_{0}\right\|+\|u\|_{L^{2}(0, T ; U)}\right) .
$$

2) Let $a(\cdot, \cdot)$ be a symmetric quadratic form satisfying $(2.2)$ and let us assume the hypotheses $(\mathrm{A}),(\mathrm{F})$. Then the equation ( $\mathrm{NCE})$ has a unique solution

$$
x \in L^{2}(0, T ; D(A)) \cap W^{1,2}(0, T ; H) \cap C([0, T] ; H),
$$

which satisfies

$$
\|x\|_{L^{2} \cap W^{1,2} \cap C} \leq C_{3}\left(1+\left\|x_{0}\right\|+\|u\|_{L^{2}(0, T ; U)}\right) .
$$


Theorem 2.3. Let $x_{0} \in V, u \in L^{2}(0, T ; U)$ and the hypotheses in 2) of Proposition 2.2 be satisfied. Then the solution $x$ of the equation (SCE) belongs to $L^{2}(0, T ; D(A)) \cap W^{1,2}(0, T ; H)$, and the mapping

$$
V \times L^{2}(0, T ; U) \ni\left(x_{0}, u\right) \mapsto x \in L^{2}(0, T ; D(A)) \cap W^{1,2}(0, T ; H)
$$

is continuous.

Proof. Let $\left(x_{0 i}, u_{i}\right) \in F \times L^{2}(0, T ; U)$, and $x_{i}$ be the solution of (SNE) with $\left(x_{0 i}, u_{i}\right)$ in place of $\left(x_{0}, u\right)$ for $i=1,2$. Then in view of $(2.7)$, we have

$$
\begin{aligned}
& \left\|x_{1}-x_{2}\right\|_{L^{2}(0, T ; D(A)) \cap W^{1,2}(0, T ; H)} \\
\leq & C_{3}\left\{\left\|x_{01}-x_{02}\right\|+\left(\left\|\partial \phi_{\epsilon}\left(x_{1}\right)-\partial \phi_{\epsilon}\left(x_{2}\right)\right\|+\left\|f\left(\cdot, x_{1}\right)-f\left(\cdot, x_{2}\right)\right\|\right)_{L^{2}(0, T ; H)}\right. \\
& \left.\left.+\left\|u_{1}-u_{2}\right\|\right)_{L^{2}(0, T ; U)}\right\} \\
\leq & C_{3}\left\{\left\|x_{01}-x_{02}\right\|+\left(\epsilon^{-1}+L\right)\left\|x_{1}-x_{2}\right\|_{L^{2}(0, T: V)}+\left\|u_{1}-u_{2}\right\|_{L^{2}(0, T ; U)}\right\} .
\end{aligned}
$$

Noting that

$$
x_{1}(t)-x_{2}(t)=x_{01}-x_{02}+\int_{0}^{t}\left(\dot{x}_{1}(s)-\dot{x}_{2}(s)\right) d s,
$$

we get

$$
\left\|x_{1}-x_{2}\right\|_{L^{2}(0, T ; H)} \leq \sqrt{T}\left\|x_{01}-x_{02}\right\|+\frac{T}{\sqrt{2}}\left\|x_{1}-x_{2}\right\|_{W^{1,2}(0, T ; H)} .
$$

Hence from (2.1) we get

$$
\begin{aligned}
& \left\|x_{1}-x_{2}\right\| L_{L^{2}(0, T ; V)} \\
\leq & C_{1}\left\|x_{1}-x_{2}\right\|_{L^{2}(0, T ; D(A))}^{1 / 2}\left\|x_{1}-x_{2}\right\|_{L^{2}(0, T ; H)}^{1 / 2} \\
\leq & C_{1}\left\|x_{1}-x_{2}\right\|_{L^{2}\left(0, T ; D\left(A_{0}\right)\right)}^{1 / 2} \\
& \times\left\{T^{1 / 4}\left\|x_{01}-x_{02}\right\|^{1 / 2}+\left(\frac{T}{\sqrt{2}}\right)^{1 / 2}\left\|x_{1}-x_{2}\right\|_{W^{1,2}(0, T ; H)}^{1 / 2}\right\} \\
\leq & C_{1} T^{1 / 4}\left\|x_{01}-x_{02}\right\|^{1 / 2}\left\|x_{1}-x_{2}\right\|_{L^{2}(0, T ; D(A))}^{1 / 2} \\
& +C_{1}\left(\frac{T}{\sqrt{2}}\right)^{1 / 2}\left\|x_{1}-x_{2}\right\|_{L^{2}(0, T ; D(A)) \cap W^{1,2}(0, T ; H)} \\
\leq & 2^{-7 / 4} C_{1}\left\|x_{01}-x_{02}\right\|+2 C_{1}\left(\frac{T}{\sqrt{2}}\right)^{1 / 2}\left\|x_{1}-x_{2}\right\|_{L^{2}(0, T ; D(A)) \cap W^{1,2}(0, T ; H)} .
\end{aligned}
$$

Combining (2.8) and (2.9) we obtain

$$
\begin{aligned}
& \left\|x_{1}-x_{2}\right\|_{L^{2}(0, T ; D(A)) \cap W^{1,2}(0, T ; H)} \\
\leq & C_{3}\left\{\left\|x_{01}-x_{02}\right\|+\left(\epsilon^{-1}+L\right)\left(2^{-7 / 4} C_{1}\left\|x_{01}-x_{02}\right\|\right.\right. \\
& \left.\left.+2 C_{1}\left(\frac{T}{\sqrt{2}}\right)^{1 / 2}\left\|x_{1}-x_{2}\right\|_{L^{2}(0, T ; D(A)) \cap W^{1,2}(0, T ; H)}\right)+\left\|u_{1}-u_{2}\right\|_{L^{2}(0, T ; U)}\right\} .
\end{aligned}
$$


Suppose that $\left(x_{0 n}, u_{n}\right) \rightarrow\left(x_{0}, u\right)$ in $V \times L^{2}(0, T ; H)$, and let $x_{n}$ and $x$ be the solutions $(\mathrm{SCE})$ with $\left(x_{0 n}, u_{n}\right)$ and $\left(x_{0}, u\right)$ respectively. Let $0<T_{1} \leq T$ be such that

$$
\left(\epsilon^{-1}+L\right) C_{1} C_{3}\left(2 T_{1}\right)^{1 / 2}<1
$$

Then by virtue of (2.10) with $T$ replaced by $T_{1}$ we see that $x_{n} \rightarrow x$ in $L^{2}\left(0, T_{1} ; D(A)\right) \cap W^{1,2}\left(0, T_{1} ; H\right) \subset C\left(\left[0, T_{1}\right] ; V\right)$. This implies that $x_{n}\left(T_{1}\right)$ $\mapsto x\left(T_{1}\right)$ in $V$. Hence the same argument shows that $x_{n} \rightarrow x$ in

$$
L^{2}\left(T_{1}, \min \left\{2 T_{1}, T\right\} ; D(A)\right) \cap W^{1,2}\left(T_{1}, \min \left\{2 T_{1}, T\right\} ; H\right) .
$$

Repeating this process we conclude that $x_{n} \rightarrow x$ in

$$
L^{2}(0, T ; D(A)) \cap W^{1,2}(0, T ; H) .
$$

\section{Preliminaries and hemivariational inequalities}

We denote $\xi \cdot \zeta=\sum_{i=1}^{N} \xi_{i} \zeta_{i}$ for $\xi=\left(\xi_{1}, \ldots, \xi_{N}\right), \zeta=\left(\zeta_{1}, \ldots, \zeta_{N}\right) \in \mathbb{R}^{N}$ and $\varepsilon \cdot \tilde{\varepsilon}=\sum_{i, j=1}^{N} \varepsilon_{i j} \tilde{\varepsilon}_{i j}$ for $\varepsilon, \tilde{\varepsilon} \in S$. Throughout this paper, we consider

$$
\begin{aligned}
& V=\left\{u \in\left(H^{1}(\Omega)\right)^{N}: u=0 \text { on } \Gamma_{1}\right\}, \quad H=\left(L^{2}(\Omega)\right)^{N}, \\
& (u, v)=\int_{\Omega} u(x) \cdot v(x) d x, \quad(u, v)_{\Gamma_{0}}=\int_{\Gamma_{0}} u(x) \cdot v(x) d \Gamma .
\end{aligned}
$$

We denote $V^{*}$ the dual space of $V,(\cdot, \cdot)$ the dual pairing between $V$ and $V^{*}$.

The norms on $V, H$ and $V^{*}$ will be denoted by $\|\cdot\|,|\cdot|$ and $\|\cdot\|_{*}$, respectively. For the sake of simplicity, we may consider

$$
\|u\|_{*} \leq|u| \leq\|u\|, \quad u \in V .
$$

We denote $\|\cdot\|_{\left(L^{2}\left(\Gamma_{0}\right)\right)^{N}}$ by $\|\cdot\|_{\Gamma_{0}}$. Let $A$ be the operator associated with a sesquilinear form $a(u, v)$ which is defined Gårding's inequality

$$
\operatorname{Re} a(u, u) \geq \omega_{1}|| u \|^{2}-\omega_{2}|u|^{2}, \quad \omega_{1}>0, \omega_{2} \geq 0, \text { for } u \in V,
$$

that is,

$$
(A u, v)=a(u, v), \quad u, v \in V .
$$

Then $A$ is a symmetric bounded linear operator from $V$ into $V^{*}$ which satisfies

$$
(A u, u) \geq \omega_{1}\|u\|^{2}-\omega_{2}|u|^{2}
$$

and its realization in $H$ which is the restriction of $A$ to

$$
D(A)=\{u \in V: A u \in H\}
$$

is also denoted by $A$. Here, we note that $D(A)$ is dense in $V$. Hence, it is also dense in $H$. We endow the domain $D(A)$ of $A$ with graph norm, that is, for $u \in D(A)$, we define $\|u\|_{D(A)}=|u|+|A u|$. So, for the brevity, we may regard that $|u| \leq\|u\| \leq\|u\|_{D(A)}$ for all $u \in V$. It is known that $-A$ generates an analytic semigroup $S(t)(t \geq 0)$ in both $H$ and $V^{*}$.

From the following inequalities

$$
\omega_{1}|| u \|^{2} \leq \operatorname{Re} a(u, u)+\omega_{2}|u|^{2} \leq C|A u||u|+\omega_{2}|u|^{2}
$$




$$
=\left(C|A u|+\omega_{2}|u|\right)|u| \leq \max \left\{C, \omega_{2}\right\}\|u\|_{D(A)}|u|,
$$

it follows that there exists a constant $C_{0}>0$ such that

$$
\|u\| \leq C_{0}\|u\|_{D(A)}^{1 / 2}|u|^{1 / 2} .
$$

So, we may regard as $V=(D(A), H)_{1 / 2,2}$ where $(D(A), H)_{1 / 2,2}$ is the real interpolation space between $D(A)$ and $H$ (see $[2,7]$ ).

If $X$ is a Banach space and $1<p<\infty, L^{p}(0, T ; X)$ is the collection of all strongly measurable functions from $(0, T)$ into $X$ the $p$-th powers whose norms are integrable and $W^{m, p}(0, T ; X)$ is the set of all functions $f$ whose derivatives $D^{\alpha} f$ up to degree $m$ in the distribution sense belong to $L^{p}(0, T ; X)$. $C^{m}([0, T] ; X)$ is the set of all $m$-times continuously differentiable functions from $[0, T]$ into $X$. Let $X$ and $Y$ be complex Banach spaces. Denote by $\mathcal{L}(X, Y)$ the set of all bounded linear operators from $X$ into $Y$. Let $\mathcal{L}(X)=\mathcal{L}(X, X)$.

From now on, we begin to introduce the regularity of solutions for the following parabolic hemivariational inequality of dynamic elasticity in the strong sense:

$$
\left\{\begin{array}{l}
x^{\prime}(t)+A x(t)-\operatorname{div} C[\varepsilon(x(t))]+\Xi(y, t)=B u(t)+f(t), \quad t \geq 0, \\
x=0 \text { on }(y, t) \in \Gamma_{1} \times(0, \infty), \\
C[\varepsilon(x(y, t))] \nu=-(\beta \cdot \nu) x(y, t) \text { on } \Gamma_{0} \times(0, \infty), \\
\Xi(y, t) \in \varphi(x(y, t)) \text { a.e. }(y, t) \in \Omega \times(0, \infty), \\
x(0)=x_{0} .
\end{array}\right.
$$

A continuous map $C$ from the space $S$ of $N \times N$ symmetric matrices into itself is defined by

$$
C[\varepsilon]=a(\operatorname{tr} \varepsilon) I+b \varepsilon, \text { for } a>0, b>0, \varepsilon \in S .
$$

It is easily known that

$$
\begin{aligned}
-(\operatorname{div} C[\varepsilon(w)], v) & =-(C[\varepsilon(w)] \nu, v)_{\Gamma_{0}}+(C[\varepsilon(w)], \varepsilon(v)) \\
& =((\beta \cdot \nu) w, v)_{\Gamma_{0}}+(C[\varepsilon(w)], \varepsilon(v)), \quad v, w \in V, \\
C\left[\varepsilon\left(w_{1}\right)\right]-C\left[\varepsilon\left(w_{2}\right)\right] & =C\left[\varepsilon\left(w_{1}-w_{2}\right)\right], \quad w_{1}, w_{2} \in V .
\end{aligned}
$$

Note that the map $C$ is linear and symmetric and it can be easily verified that the tensor $C$ satisfies the condition

$$
\lambda_{0}|\varepsilon|^{2} \leq C[\varepsilon] \cdot \varepsilon \leq \lambda_{1}|\varepsilon|^{2}, \quad \varepsilon \in S \text { for some } \lambda_{0}, \lambda_{1}>0 .
$$

Let $\lambda$ be the smallest positive constant such that

$$
\|v\|^{2} \leq \lambda\|\nabla v\|^{2} \quad \text { for all } v \in V .
$$

Simple calculations and Korn's inequality yield that

$$
\lambda_{2}|\nabla u|^{2} \leq|\varepsilon(u)|^{2} \leq \lambda_{3}|\nabla u|^{2},
$$

and hence $|\varepsilon(u)|$ is equivalent to the $\left(H^{1}(\Omega)\right)^{N}$ norm on $V$.

Now, we formulate the following assumptions: 
(Hb) Let $b_{i}(i=1, \ldots, N): \mathbb{R} \rightarrow \mathbb{R}$ be a locally bounded function verifying

$$
\left|b_{i}(s)\right| \leq \mu_{i}|s| \text { for } s \in \mathbb{R},
$$

where $\mu_{i}>0$. We denote

$$
\widetilde{\mu}=\max \left\{\mu_{1}, \ldots, \mu_{N}\right\} .
$$

The multi-valued function $\varphi_{i}: \mathbb{R} \rightarrow 2^{\mathbb{R}}$ is obtained by filling in jumps of a function $b_{i}: \mathbb{R} \rightarrow \mathbb{R}$ by means of the functions $\underline{b_{i}}{ }^{\epsilon},{\overline{b_{i}}}^{\epsilon}, \underline{b_{i}}, \overline{b_{i}}: \mathbb{R} \rightarrow \mathbb{R}$ as follows.

$$
\begin{aligned}
& {\underline{b_{i}}}^{\epsilon}(s)=\operatorname{ess} \inf _{|\tau-s| \leq \epsilon} b_{i}(\tau),{\overline{b_{i}}}^{\epsilon}(s)=\operatorname{ess} \sup _{|\tau-s| \leq \epsilon} b_{i}(\tau), \\
& \underline{b_{i}}(s)=\lim _{\epsilon \rightarrow 0^{+}} \underline{b_{i}}{ }^{\epsilon}(s), \overline{b_{i}}(s)=\lim _{\epsilon \rightarrow 0^{+}} \overline{b_{i}}{ }^{\epsilon}(s), \\
& \varphi_{i}(s)=\left[\underline{b_{i}}(s), \overline{b_{i}}(s)\right] .
\end{aligned}
$$

We denote $b(\xi):=\left(b_{1}\left(\xi_{1}\right), \ldots, b_{N}\left(\xi_{N}\right)\right), \varphi(\xi):=\left(\varphi_{1}\left(\xi_{1}\right), \ldots, \varphi_{N}\left(\xi_{N}\right)\right)$ for $\widetilde{\xi}=$ $\left(\xi_{1}, \ldots, \xi_{N}\right) \in \mathbb{R}^{N}$. We shall need a regularization of $b_{i}$ defined by

$$
b_{i}^{n}(s)=n \int_{-\infty}^{\infty} b_{i}(s-\tau) \rho(n \tau) d \tau
$$

where $\rho \in C_{0}^{\infty}((-1,1)), \rho \geq 0$ and $\int_{-1}^{1} \rho(\tau) d \tau=1$. It is easy to show that $b_{i}^{n}$ is continuous for all $n \in \mathbb{N}$ and $\underline{b_{i}}{ }^{\epsilon}, \overline{b_{i}}{ }^{\epsilon}, b_{i}, \overline{b_{i}}, b_{i}^{n}$ satisfy the same condition (Hb) with possibly different constants if $b_{i}$ satisfies $(\mathrm{Hb})$. It is also known that $b_{i}^{n}(s)$ is locally Lipschitz continuous in $s$, i.e., for any $r>0$, there exists a number $L_{i}(r)>0$ such that

$$
\left|b_{i}^{n}\left(s_{1}\right)-b_{i}^{n}\left(s_{2}\right)\right| \leq L_{i}(r)\left|s_{1}-s_{2}\right|
$$

holds for all $s_{1}, s_{2} \in \mathbb{R}$ with $\left|s_{1}\right|<r,\left|s_{2}\right|<r$. We denote

$$
L(r)=\max \left\{L_{1}(r), \ldots, L_{N}(r)\right\} .
$$

Here, we remark that $b^{n}(s)$ is $C^{\infty}$ with Fréchet derivative $D b^{n}(s)$ such that $D b^{n}(s) \leq L(r)$.

From now on, we establish the following results on the solvability of the following equation.

(HIE-1)

$$
\left\{\begin{array}{l}
x^{\prime}(t)+A x(t)-\operatorname{div} C[\varepsilon(x(t))]=-b^{n}(x(t))+B u(t)+f(t), t \geq 0 \\
x=0 \text { on } \Gamma_{1} \times(0, \infty) \\
C[\varepsilon(x(y, t))] \nu=-(\beta \cdot \nu) x(y, t) \text { on } \Gamma_{0} \times(0, \infty) \\
x(0)=x_{0}
\end{array}\right.
$$

Referring to the result by Park et al. [21], the wellposedness of (HIE-1) can be given as follows.

Proposition 3.1. Suppose that the assumptions stated above are satisfied. Then the following properties hold: 
Let $\left(x_{0}, f\right) \in H \times L^{2}\left(0, T ; V^{*}\right)$ and $B \in \mathcal{L}\left(Y, V^{*}\right)$. Then there exists a unique solution $x$ of (1.1) belonging to

$$
L^{2}(0, T ; V) \cap W^{1,2}\left(0, T ; V^{*}\right) \subset C([0, T] ; H)
$$

and satisfying

(2.8) $\|x\|_{L^{2}(0, T ; V) \cap W^{1,2}\left(0, T ; V^{*}\right)} \leq C_{1}\left(\left|x_{0}\right|+\|u\|_{L^{2}(0, T ; Y)}+\|f\|_{L^{2}\left(0, T ; V^{*}\right)}\right)$, where $C_{1}$ is a constant depending on $T$.

Let $\hat{a}(u, v)$ be a bounded sesquilinear form defined in $V \times V$ by

$$
\hat{a}(u, v)=(A u, v)-(\operatorname{div} C[\varepsilon(u)], v), \quad u, v \in V .
$$

Noting that by $(2.3)$

$$
-(\operatorname{div} C[\varepsilon(u)], u)=(C[\varepsilon(u)], \varepsilon(u))+((\beta \cdot \nu) u, u)_{\Gamma_{0}},
$$

and by (2.5), (2.7) and (1.2),

$$
\lambda_{0} \lambda_{2}\|u\|^{2} \leq(C[\varepsilon(u)], \varepsilon(u)), \quad \alpha\|u\|_{\Gamma_{0}}^{2} \leq((\beta \cdot \nu) u, u)_{\Gamma_{0}},
$$

it follows that there exist $\hat{\omega}_{1}>0$ and $\hat{\omega}_{2} \geq 0$ such that

$$
\operatorname{Re} \hat{a}(u, u) \geq \hat{\omega}_{1}|| u \|^{2}-\hat{\omega}_{2}|u|^{2} \quad \text { for } u \in V \text {. }
$$

Let $\hat{A}$ be the operator associated with this sesquilinear form:

$$
(\hat{A} u, v)=\hat{a}(u, v), \quad u, v \in V .
$$

Then $\hat{A}$ is also a symmetric continuous linear operator from $V$ into $V^{*}$ which satisfies

$$
(\hat{A} u, u) \geq \hat{\omega}_{1}|| u \|^{2}-\hat{\omega}_{2}|u|^{2} .
$$

So we know that $-\hat{A}$ generates an analytic semigroup $\hat{S}(t)(t \geq 0)$ in both $H$ and $V^{*}$. Hence, by applying Proposition 2.1 to the regularity for the solution of the equation:

$$
\left\{\begin{array}{l}
x^{\prime}(t)+\hat{A} x(t)=-b^{n}(x(t))+B u(t)+f(t), \quad t>0, \\
x=0 \text { on } \Gamma_{1} \times(0, \infty), \\
C[\varepsilon(x(y, t))] \nu=-(\beta \cdot \nu) x(y, t) \text { on } \Gamma_{0} \times(0, \infty), \\
x(y, 0)=x_{0}(y), \quad y \in \Omega,
\end{array}\right.
$$

in the space $H$, we can obtain a unique solution $x$ belonging to

$$
L^{2}(0, T ; V) \cap W^{1,2}\left(0, T ; V^{*}\right) \subset C([0, T] ; H)
$$

and satisfying the norm estimate (2.8).

We set

$$
F(x)=\int_{0}^{1}-D b^{n}(r x) d r, \quad x \in V .
$$

Then, using the assumption (Hb-1), we see the following properties:

$$
-b^{n}(x)=F(x) x-b^{n}(0),
$$




$$
\|F(x(t))\|_{\mathcal{L}(V, H)} \leq L, \quad x \in C([0, T] ; V) .
$$

Therefore, the problem (HIE-1) can be rewritten as

$$
x(t)=\hat{S}(t) x_{0}+\int_{0}^{t} \hat{S}(t-s)\left[F(x(s)) x(s)+B u(s)-b^{n}(0)+f(s)\right] d s,
$$

or, by perturbations of semigroup theory,

$$
x(t)=Q(t ; F) x_{0}+\int_{0}^{t} Q(t-s ; F)\left[B u(s)-b^{n}(0)+f(s)\right] d s,
$$

where

$Q(t-s ; F) y=S(t-s) y+\int_{s}^{t} S(t-r) F(x(r)) Q(r-s ; F) y d r, \quad 0 \leq s \leq t \leq T$.

Remark 3.2. 1) It is easily seen that

$$
H=\left\{x \in V^{*}: \int_{0}^{T}\left\|A e^{t A} x\right\|_{*}^{2} d t<\infty\right\}
$$

for the time $T>0$. Therefore, in terms of the intermediate theory we can see that

$$
\left(V, V^{*}\right)_{1 / 2,2}=H \text {. }
$$

In terms of Proposition 2.1, we remark that if $x_{0} \in V=(D(A), H)_{1 / 2,2}$, $f \in L^{2}(0, T ; H)$ and $B \in \mathcal{L}(Y, H)$ for any $T>0$, then the solution $x$ of (HIE-1) exists and is unique in

$$
x \in L^{2}(0, T ; D(A)) \cap W^{1,2}(0, T ; H) \subset C([0, T] ; V) .
$$

Furthermore, there exists a constant $C_{1}$ depending on $T$ such that

$$
\|x\|_{L^{2} \cap W^{1,2}} \leq C_{1}\left(1+\left\|x_{0}\right\|+\|u\|_{L^{2}(0, T ; Y)}+\|f\|_{L^{2}(0, T ; H)}\right) .
$$

2) Let $x$ be a solution of (HIE-1) and $x \in B_{r}=\left\{v \in L^{2}(0, T ; V):\|v\| \leq r\right\}$. Then the following inequality holds: for any $0<t \leq T$,

$$
\begin{gathered}
\|x\|_{C([0, t] ; H) \cap L^{2}(0, t ; V) \cap L^{2}\left(0, t ;\left(L^{2}\left(\Gamma_{0}\right)\right)^{N}\right)}^{2} \\
\leq c_{1}^{-1}\left(\frac{1}{2}\left|x_{0}\right|^{2}+\|u\|_{L^{2}(0, t ; Y)}^{2}+\|f\|_{L^{2}(0, t ; H)}^{2}\right),
\end{gathered}
$$

where $c_{1}=\min \left\{1 / 2, \alpha, \omega_{1}+c_{0}\right\} e^{-\left(\omega_{2}+L(r)+1\right) t}$. In fact, we remark that from (2.4) and (2.5), it follows that there is a constant $c_{0}>0$ such that

(2.12) $\quad c_{0}\left\|x_{1}(t)-x_{2}(t)\right\|^{2} \leq\left(C\left[\varepsilon\left(x_{1}(t)\right)\right]-C\left[\varepsilon\left(x_{2}(t)\right)\right], \varepsilon\left(x_{1}(t)\right)-\varepsilon\left(x_{2}(t)\right)\right)$.

Multiplying $x(t)$ on both sides of (HIE-1), we get

$$
\begin{aligned}
& \left(x^{\prime}(t), x(t)\right)+(A x(t), x(t))+(C[\varepsilon(x(t))], \varepsilon(x(t)))+((\beta \cdot \nu) x(t), x(t)) \\
& +\left(b^{n}(x(t)), x(t)\right)=(B u(t), x(t))+(f(t), x(t)),
\end{aligned}
$$

and integrating this over $(0, t)$, by $(2.1),(2.12)$ and (Hb-1), we have

$$
\frac{1}{2}|x(t)|^{2}+\alpha \int_{0}^{t}\|x(\tau)\|_{\Gamma_{0}}^{2} d \tau+\left(\omega_{1}+c_{0}\right) \int_{0}^{t}\|x(\tau)\|^{2} d \tau
$$




$$
\leq \frac{1}{2}\left|x_{0}\right|^{2}+\left(\omega_{2}+L(r)\right) \int_{0}^{t}|x(\tau)|^{2} d \tau+\int_{0}^{t}\left\{|B u(\tau)+f(\tau)|^{2}+|x(\tau)|^{2}\right\} d \tau .
$$

Applying Gronwall Lemma, we obtain (2.11).

The following results for the continuity of solution map in the sense of Proposition 2.1 and Remark 2.1 follow immediately from Theorem 3.7 of [21].

Proposition 3.3. Let the assumption $(\mathrm{Hb})$ be satisfied.

1) If $\left(x_{0}, u, f\right) \in V \times L^{2}(0, T ; Y) \times L^{2}(0, T ; H)$ and $B \in \mathcal{L}(Y, H)$, then the solution $x$ of the equation (HIE) belongs to $x \in L^{2}(0, T ; D(A)) \cap C([0, T] ; V)$ and the mapping

$$
V \times L^{2}(0, T ; Y) \ni\left(x_{0}, u\right) \mapsto x \in L^{2}(0, T ; D(A)) \cap C([0, T] ; V)
$$

is continuous.

2) If $\left(x_{0}, u, f\right) \in H \times L^{2}(0, T ; Y) \times L^{2}\left(0, T ; V^{*}\right)$ and $B \in \mathcal{L}\left(Y, V^{*}\right)$. Then the solution $x$ of the equation (HIE) belongs to $x \in L^{2}(0, T ; V) \cap C([0, T] ; H)$ and the mapping

$$
H \times L^{2}(0, T ; Y) \ni\left(x_{0}, u\right) \mapsto x \in L^{2}(0, T ; V) \cap C([0, T] ; H)
$$

is continuous.

\section{Optimal control problems}

In this section we study the optimal control problems for quadratic cost function in framework of Lions [12]. In what follows we assume that the embedding $D(A) \subset V \subset H$ is compact.

Let $Y$ be another Hilbert space, and $B$ be an bounded linear operator from $Y$ into $H$, i.e.,

$$
B \in \mathcal{L}(Y, H)
$$

which is called a controller.

Let the solution spaces $\mathcal{W}$ and $\mathcal{W}_{1}$ of (1.1) of strong solutions are defined by

$$
\begin{aligned}
& \mathcal{W}=L^{2}(0, T ; V) \cap W^{1,2}\left(0, T ; V^{*}\right) \subset C([0, T] ; H), \\
& \mathcal{W}_{1}=L^{2}(0, T ; D(A)) \cap W^{1,2}(0, T ; H) \subset C([0, T] ; V),
\end{aligned}
$$

respectively, as stated in Proposition 2.1 and Remark 2.1. By virtue of Proposition 2.1, we can define uniquely the solution map $u \mapsto x(u)$ of $L^{2}(0, T ; Y)$ into $\mathcal{W}$. We shall call the solution $x(u)$ the state of the control system (1.1).

Let $\mathcal{Y}=L^{2}(0, T ; Y)$ be a Hilbert space of control variables. Choose a bounded subset $U$ of $Y$. Let us define an admissible control $U_{a d}$ as

$$
\begin{aligned}
U_{a d}= & \{u \in \mathcal{Y}: u \text { is strongly measurable function satisfying } \\
& u(t) \in U \text { for almost all } t\} .
\end{aligned}
$$

Let $M$ be a Hilbert space of observation variables. The observation of state is assumed to be given by

$$
z(u)=\mathcal{C} x(u), \quad \mathcal{C} \in \mathcal{L}\left(\mathcal{W}_{1}, M\right),
$$


where $\mathcal{C}$ is an operator called the observe.

Let $z_{d} \in M$. Suppose that there exists no admissible control which satisfies $\mathcal{C} x(u)=z_{d}$. The quadratic cost function associated with the control system (1.1) is given by

$$
J(v)=\left\|\mathcal{C} x(v)-z_{d}\right\|_{M}^{2}+(R v, v) \mathcal{Y} \quad \text { for } v \in U_{a d},
$$

where $z_{d} \in M$ is a desire value of $x(v)$ and $R \in \mathcal{L}(\mathcal{Y}, \mathcal{Y})$ is symmetric and positive, i.e.,

$$
(R v, v)_{\mathcal{Y}}=(v, R v)_{\mathcal{Y}} \geq d\|v\|_{\mathcal{Y}}^{2}
$$

for some $d>0$.

An element $u \in U_{a d}$ which attains minimum of $J(v)$ over $U_{a d}$ is called an optimal control for the cost function (3.3).

Remark 4.1. We consider the following two types of observation $\mathcal{C}$ of distributive and terminal values (see $[9,10])$.

1) We take $M=L^{2}(\Omega \times(0, T)) \times L^{2}(\Omega)$ and $\mathcal{C} \in \mathcal{L}(\mathcal{W}, M)$ and observe

$$
z(v)=\mathcal{C} x(v)=(x(v ; \cdot), x(v, T)) \in L^{2}(\Omega \times(0, T)) \times L^{2}(\Omega) ;
$$

2) We take $M=L^{2}(\Omega \times(0, T))$ and $\mathcal{C} \in \mathcal{L}(\mathcal{W}, M)$ and observe

$$
z(v)=\mathcal{C} x(v)=x^{\prime}(v ; \cdot) \in L^{2}(\Omega \times(0, T)) .
$$

The above observations are meaningful in view of the regularity of the equation (1.1) by Proposition 2.1.

Theorem 4.2. Let the assumption $(\mathrm{Hb})$ be satisfied.

1) Assume that $\left(x_{0}, f\right) \in H \times L^{2}\left(0, T ; V^{*}\right)$ and $B \in \mathcal{L}\left(Y, V^{*}\right)$. Then the mapping $u \mapsto x(u)$ is compact from $L^{2}(0, T ; Y)$ to $L^{2}(0, T ; H)$.

2) Assume that $\left(x_{0}, f\right) \in V \times L^{2}(0, T ; H)$ and $B \in \mathcal{L}(Y, H)$. Then the mapping $u \mapsto x(u)$ is compact from $L^{2}(0, T ; Y)$ to $L^{2}(0, T ; V)$.

Proof. 1) We define the solution mapping $S$ from $L^{2}(0, T ; Y)$ to $L^{2}(0, T ; H)$ by

$$
S u=x(u), \quad u \in L^{2}(0, T ; Y) .
$$

In virtue of Proposition 2.1, we have

$\|S u\|_{L^{2}(0, T ; V) \cap W^{1,2}\left(0, T ; V^{*}\right)}=\|x(u)\| \leq C_{1}\left\{\left|x_{0}\right|+\|u\|_{L^{2}(0, T ; Y)}+\|f\|_{L^{2}\left(0, T ; V^{*}\right)}\right\}$.

Hence if $u$ is bounded in $L^{2}(0, T ; Y)$, then so is $x(u)$ in $L^{2}(0, T ; V) \cap W^{1,2}(0, T$; $\left.V^{*}\right)$. Since $V$ is compactly embedded in $H$ by assumption, the embedding $L^{2}(0, T ; V) \cap W^{1,2}\left(0, T ; V^{*}\right) \subset L^{2}(0, T ; H)$ is also compact in view of Theorem 2 of Aubin [1]. Hence, the mapping $u \mapsto S u=x(u)$ is compact from $L^{2}(0, T ; Y)$ to $L^{2}(0, T ; H)$.

2) If $D(A)$ is compactly embedded in $V$ by assumption, the embedding

$$
L^{2}(0, T ; D(A)) \cap W^{1,2}(0, T ; H) \subset L^{2}(0, T ; V)
$$

is compact. Hence, the proof of 2) is complete. 
As indicated in Introduction we need to show the existence of an optimal control and to give the characterizations of them. The existence of an optimal control $u$ for the cost function (3.3) can be stated by the following theorem.

Theorem 4.3. Let the assumption $(\mathrm{Hb})$ be satisfied and $x_{0} \in \overline{D(\phi)} \cap V$. Then there exists at least one optimal control u for the control problem (1.1) associated with the cost function (3.2), i.e., there exists $u \in U_{\text {ad }}$ such that

$$
J(u)=\inf _{v \in U_{a d}} J(v):=J .
$$

Proof. Since $U_{a d}$ is non-empty and $J$ is bounded from below, there is a sequence $\left\{u_{n}\right\} \subset U_{a d}$ such that minimizing sequence for the problem (3.5), which satisfies

$$
\inf _{v \in U_{a d}} J(v)=\lim _{n \rightarrow \infty} J\left(u_{n}\right)=m .
$$

Obviously, $\left\{J\left(u_{n}\right)\right\}$ is bounded. Hence by (3.4) there is a positive constant $K_{0}$ such that

$$
d\left\|u_{n}\right\|_{\mathcal{Y}}^{2} \leq\left(R u_{n}, u_{n}\right) \mathcal{Y} \leq J\left(u_{n}\right) \leq K_{0} .
$$

This show that $\left\{u_{n}\right\}$ is bounded in $U_{a d}$. So we can extract a subsequence (denote again by $\left\{u_{n}\right\}$ ) of $\left\{u_{n}\right\}$ and find a $u \in U_{a d}$ such that

$$
u_{n} \rightarrow u \text { weakly in } U_{a d} .
$$

Let $x_{n}=x\left(u_{n}\right)$ be the solution of the following equation corresponding to $u_{n}$ :

$$
\left\{\begin{array}{l}
x_{n}^{\prime}(t)+A x_{n}(t)-\operatorname{div} C\left[\varepsilon\left(x_{n}(t)\right)\right]=-b^{n}\left(x_{n}(t)\right)+B u_{n}(t)+f(t), \quad t \geq 0, \\
x_{n}=0 \text { on } \Gamma_{1} \times(0, \infty), \\
C\left[\varepsilon\left(x_{n}(y, t)\right)\right] \nu=-(\beta \cdot \nu) x_{n}(y, t) \text { on } \Gamma_{0} \times(0, \infty), \\
x_{n}(0)=x_{0} .
\end{array}\right.
$$

By (2.8) and the above equation, we know $\left\{x_{n}\right\}$ and $\left\{x_{n}^{\prime}\right\}$ are bounded in $L^{2}(0, T ; V)$ and $L^{2}\left(0, T ; V^{*}\right)$, respectively. Therefore, by the extraction theorem of Rellich's, we can find a subsequence of $\left\{x_{n}\right\}$, say again $\left\{x_{n}\right\}$ and find $x$ such that

$$
\begin{aligned}
& x_{n}(\cdot) \rightarrow x(\cdot) \text { weakly in } L^{2}(0, T ; V) \cap C([0, T] ; H), \\
& b^{n}\left(x_{n}\right) \rightarrow \Xi \text { weakly in } C([0, T] ; H) .
\end{aligned}
$$

and

$$
x_{n}^{\prime} \rightarrow x^{\prime} \text { weakly in } L^{2}\left(0, T ; V^{*}\right) .
$$

Here, we remark that as seen in Theorem 3.5 of [21], we know that $\Xi(y, t) \in$ $\varphi(x(y, t))$ a.e. in $\Omega \times(0, T)$. By Theorem 3.1, we know that

$$
x_{n}(\cdot) \rightarrow x(\cdot) \text { strongly in } L^{2}(0, T ; V) .
$$

From (Hb) it follows that

$$
b^{n}\left(x_{n}\right) \rightarrow \Xi \text { strongly in } L^{2}(0, T ; H) .
$$


By the boundedness of $A$ we have

$$
A x_{n} \rightarrow A x \text { strongly in } L^{2}\left(0, T ; V^{*}\right) .
$$

Since $b^{n}(x)$ is locally Lipschitz continuous in $x$, from (3.6)-(3.8) it follows that $\dot{x}_{n}(t) \rightarrow-A x(t)+\operatorname{div} C[\varepsilon(x(t))]-\Xi(x(t))+B u(t)+f(t)$, weakly in $L^{2}\left(0, T ; V^{*}\right)$.

By letting $n \rightarrow \infty$ and using the convergence results above, we have proved that $x(t)$ satisfies a.e. on $(0, T)$ the following equation:

$$
\left\{\begin{array}{l}
x^{\prime}(t)+A x(t)-\operatorname{div} C[\varepsilon(x(t))]=-\Xi(x(t))+B u(t)+f(t), t \geq 0, \\
x=0 \text { on } \Gamma_{1} \times(0, \infty), \\
C[\varepsilon(x(y, t))] \nu=-(\beta \cdot \nu) x(y, t), \text { on } \Gamma_{0} \times(0, \infty), \\
x(0)=x_{0} .
\end{array}\right.
$$

Since $\mathcal{C}$ is continuous on $L^{2}(0, T ; D(A)) \cap W^{1,2}(0, T ; H)$ and $\|\cdot\|_{M}$ is lower semicontinuous, it holds that

$$
\left\|\mathcal{C} x(u)-z_{d}\right\|_{M} \leq \liminf _{n \rightarrow \infty}\left\|\mathcal{C} x\left(u_{n}\right)-z_{d}\right\|_{M} .
$$

It is also clear from $\liminf _{n \rightarrow \infty}\left\|R^{1 / 2} u_{n}\right\|_{\mathcal{Y}} \geq\left\|R^{1 / 2} u\right\|_{\mathcal{Y}}$ that

$$
\liminf _{n \rightarrow \infty}\left(R u_{n}, u_{n}\right) \mathcal{Y} \geq(R u, u) \mathcal{Y}
$$

Thus,

$$
m=\lim _{n \rightarrow \infty} J\left(u_{n}\right) \geq J(u) .
$$

But since $J(u) \geq m$ by definition, we conclude $u \in U_{a d}$ is the desired optimal control.

\section{Necessary optimal condition}

In this section we shall characterize the optimal controls by giving necessary conditions for optimality. For this it is necessary to write down the necessary optimal condition

$$
D J(u)(v-u) \geq 0, \quad v \in L^{2}(0, T ; Y)
$$

and to analyze (4.1) in view of the proper adjoint state system, where $D J(u)$ denote the Gâteaux derivative of $J(v)$ at $v=u$. Therefore, we have to prove that the solution mapping $v \mapsto x(v)$ is Gâteaux differentiable at $v=u$. Here we note that from Proposition 2.2 it follows immediately that

$$
\lim _{\lambda \rightarrow 0} x(u+\lambda w)=x(u) \text { strongly in } L^{2}(0, T ; V) \cap C([0, T] ; H) .
$$

The solution map $v \mapsto x(v)$ of $L^{2}(0, T ; Y)$ into $L^{2}(0, T ; V) \cap C([0, T] ; H)$ is said to be Gâteaux differentiable at $v=u$ if for any $w \in L^{2}(0, T ; Y)$ there exist a $D x(u) \in \mathcal{L}\left(L^{2}(0, T ; Y), L^{2}(0, T ; V) \cap C([0, T] ; H)\right)$ such that

$$
\left\|\frac{1}{\lambda}(x(u+\lambda w)-x(u))-D x(u) w\right\| \rightarrow 0 \quad \text { as } \quad \lambda \rightarrow 0 .
$$


The operator $D x(u)$ denotes the Gâteaux derivative of $x(u)$ at $v=u$ and the function $D x(u) w \in L^{2}(0, T ; V) \cap C([0, T] ; H)$ is called the Gâteaux derivative in the direction $w \in L^{2}(0, T ; Y)$, which plays an important part in the nonlinear optimal control problems.

Now, we introduce the smoothing system corresponding to (1.1) as follows. (4.3)

$$
\left\{\begin{array}{l}
x^{\prime}(t)+A x(t)-\operatorname{div} C[\varepsilon(x(t))]=-b^{n}(x(t))+B u(t)+f(t), t \geq 0, n \in \mathbb{N}, \\
x=0 \text { on } \Gamma_{1} \times(0, \infty), \\
C[\varepsilon(x(y, t))] \nu=-(\beta \cdot \nu) x(y, t) \text { on } \Gamma_{0} \times(0, \infty), \\
x(0)=x_{0} .
\end{array}\right.
$$

Theorem 5.1. Let the assumption $(\mathrm{Hb})$ be satisfied and let $u \in \mathcal{U}_{\text {ad }}$ be an optimal control for the cost function $J$ in (3.3). Then the following inequality:

$$
\left\langle\mathcal{C}^{*} \Lambda_{M}\left(\mathcal{C} x(u)-z_{d}\right), y\right\rangle_{\mathcal{W}_{1 *}, \mathcal{W}_{1}}+(R u, v-u)_{L^{2}(0, T ; Y)} \geq 0, \quad \forall v \in \mathcal{U}_{a d}
$$

holds, where $y=D x(u)(v-u) \in \mathcal{W}_{1}$ is a unique solution of the following equation:

$$
\left\{\begin{array}{l}
y^{\prime}(t)+A y(t)-\operatorname{div} C[\varepsilon(y)]=-D b^{n}(x) y(t)+B(u-v)(t), 0<t \leq T \\
y(0)=0
\end{array}\right.
$$

Proof. We set $w=v-u$. Let $\lambda \in(-1,1), \lambda \neq 0$. We set

$$
y=\lim _{\lambda \rightarrow 0} \lambda^{-1}(x(u+\lambda w)-x(u))=D x(u) w .
$$

From (4.3), we have

$$
\begin{aligned}
& x^{\prime}(u+\lambda w)-x^{\prime}(u)+A(x(u+\lambda w)-x(u))-\operatorname{div} C[\varepsilon(x(u+\lambda w)-x(u))] \\
= & -b^{n}(x(u+\lambda w))+b^{n}(x(u))+\lambda B w .
\end{aligned}
$$

It is easily seen that

$$
y=D x(u) w=x(v)-x(u)
$$

and

$$
\begin{aligned}
& \lim _{\lambda \rightarrow 0} \frac{1}{\lambda}\{\operatorname{div} C[\varepsilon(x(u+\lambda w)-x(u))]\}=\operatorname{div} C[\varepsilon(y)], \\
& \lim _{\lambda \rightarrow 0} \frac{1}{\lambda}\left\{b^{n}(x(u+\lambda w))-b^{n}(x(u))\right\}=D b^{n}(x) y .
\end{aligned}
$$

Thus, from (4.6) it follows that $y=D x(u)(v-u)$ satisfies (4.5) and the cost function $J(v)$ is Gâteaux differentiable at $v=u$ in the direction $w=v-u$. The optimal condition (4.1) is rewritten by

$$
\begin{aligned}
& \left(\mathcal{C} x(u)-z_{d}, \mathcal{C} y\right)_{M}+(R u, v-u)_{L^{2}(0, T ; Y)} \\
= & \left\langle\mathcal{C}^{*} \Lambda_{M}\left(\mathcal{C} x(u)-z_{d}\right), y\right\rangle_{\mathcal{W}_{1}, \mathcal{W}_{1}}+(R u, v-u)_{L^{2}(0, T ; Y)} \geq 0, \quad \forall v \in U_{a d} .
\end{aligned}
$$


With every control $u \in L^{2}(0, T ; Y)$, we consider the following distributional cost function expressed by

$$
J_{1}(u)=\int_{0}^{T}\left\|\mathcal{C} x_{u}(t)-z_{d}(t)\right\|_{X}^{2} d t+\int_{0}^{T}(R u(t), u(t)) d t,
$$

where the operator $\mathcal{C}$ is bounded from $H$ to another Hilbert space $X$ and $z_{d} \in L^{2}(0, T ; X)$. Finally we are given $R$ is self adjoint and positive definite:

$$
R \in \mathcal{L}(Y), \quad \text { and } \quad(R u, u) \geq c\|u\|^{2}, \quad c>0 .
$$

Let $x_{u}(t)$ stand for solution of (1.1) associated with the control $u \in L^{2}(0, T ; Y)$. Let $U_{a d}$ be a closed convex subset of $L^{2}(0, T ; Y)$.

Theorem 5.2. Let the operators $\mathcal{C}$ and $R$ satisfy the conditions mentioned above. Then there exists a unique element $u \in U_{\text {ad }}$ such that

$$
J_{1}(u)=\inf _{v \in U_{a d}} J_{1}(v)
$$

Furthermore, it holds the following inequality:

$$
\int_{0}^{T}\left(\Lambda_{Y}^{-1} B^{*} p_{u}(t)+R u(t),(v-u)(t)\right)_{Y} d t \geq 0, \quad \forall v \in U_{a d}
$$

holds, where $\Lambda_{Y}$ is the canonical isomorphism $Y$ into $Y^{*}$ and $p_{u}$ satisfies the following equation:

$$
\left\{\begin{array}{l}
p_{u}^{\prime}(t)-A^{*} p_{u}(t)-\operatorname{div} \varepsilon^{*} C^{*}\left(p_{u}\right)-D b^{n}(x)^{*} p_{u}(t) \\
=-\mathcal{C}^{*} \Lambda_{X}\left(\mathcal{C} x_{u}(t)-z_{d}(t)\right) \quad \text { for } 0<t \leq T \\
P_{u}(T)=0
\end{array}\right.
$$

Proof. Let $x_{u}$ be a solution of (1.1) associated with the control $u$. Then it holds that

$$
\begin{aligned}
J_{1}(v) & =\int_{0}^{T}\left\|\mathcal{C} x_{v}(t)-z_{d}(t)\right\|^{2} d t+\int_{0}^{T}(R v(t), v(t)) d t \\
& =\int_{0}^{T}\left\|\mathcal{C}\left(x_{v}(t)-x(t)\right)+\mathcal{C} x(t)-z_{d}(t)\right\|^{2} d t+\int_{0}^{T}(R v(t), v(t)) d t \\
& =\pi(v, v)-2 L(v)+\int_{0}^{T}\left\|z_{d}(t)-\mathcal{C} x(t)\right\|_{X}^{2} d t
\end{aligned}
$$

where

$$
\begin{aligned}
\pi(u, v)= & \int_{0}^{T}\left(\mathcal{C}\left(x_{u}(t)-x(t)\right), \mathcal{C}\left(x_{v}(t)-x(t)\right)\right)_{X} d t \\
& \quad+\int_{0}^{T}(R u(t), v(t))_{Y} d t \\
L(v)= & \int_{0}^{T}\left(z_{d}(t)-\mathcal{C} x(t), \mathcal{C}\left(x_{v}(t)-x(t)\right)\right)_{X} d t .
\end{aligned}
$$


The form $\pi(u, v)$ is a continuous bilinear form in $L^{2}(0, T ; Y) \times L^{2}(0, T ; Y)$ and from assumption of the positive definite of the operator $R$, we have

$$
\pi(v, v) \geq c\|v\|^{2}, \quad v \in L^{2}(0, T ; Y) .
$$

Therefore, by Theorem 3.1 (in virtue of Theorem 1.1 of Chapter 1 in [12]) there exists a unique $u \in U_{a d}$ such that (4.7). If $u$ is an optimal control, similarly for (4.4), (4.1) is equivalent to

$$
\int_{0}^{T}\left(\mathcal{C}^{*} \Lambda_{X}\left(\mathcal{C} x_{u}(t)-z_{d}(t)\right), y(t)\right) d t+\int_{0}^{T}(R u(t),(v-u)(t))_{Y} d t \geq 0 .
$$

Now we formulate the adjoint system to describe the optimal condition:

$$
\left\{\begin{array}{l}
p_{u}^{\prime}(t)-A^{*} p_{u}(t)-\operatorname{div} \varepsilon^{*} \mathcal{C}^{*}\left(p_{u}(t)\right)-D b^{n}(x)^{*} p_{u}(t) \\
=-\mathcal{C}^{*} \Lambda_{X}\left(\mathcal{C} x_{u}(t)-z_{d}(t)\right) \text { for } 0<t \leq T \\
P_{u}(T)=0
\end{array}\right.
$$

Taking into account the regularity result of Proposition 2.1 and the observation conditions, we can assert that (4.11) admits a unique weak solution $p_{u}$ reversing the direction of time $t \rightarrow T-t$ by referring to the wellposedness result of Dautray and Lions [8, pp. 558-570].

We multiply both sides of equation (4.11) by $y(t)$ of (4.5) and integrate it over $[0, T]$. Then we have

$$
\begin{aligned}
& \int_{0}^{T}\left(\mathcal{C}^{*} \Lambda_{X}\left(\mathcal{C} x_{u}(t)-z_{d}(t)\right), y(t)\right) d t \\
= & -\int_{0}^{T}\left(p_{u}^{\prime}(t), y(t)\right) d t+\int_{0}^{T}\left(A^{*} p_{u}(t), y(t)\right) d t+\int_{0}^{T}\left(\operatorname{div} \varepsilon^{*} C^{*}\left(p_{u}(t)\right), y(t)\right) d t \\
& +\int_{0}^{T}\left(D b^{n}(x)^{*} p_{u}(t), y(t)\right) d t .
\end{aligned}
$$

By the initial value condition of $y$ and the terminal value condition of $p_{u}$, the left hand side of (4.12) yields

$$
\begin{aligned}
& \left(p_{u}(T), y(T)\right)-\left(p_{u}(0), y(0)\right)+\int_{0}^{T}\left(p_{u}(t), y^{\prime}(t)\right) d t+\int_{0}^{T}\left(p_{u}(t), A y(t)\right) d t \\
& -\int_{0}^{T}\left(p_{u}(t), \operatorname{div} C[\varepsilon(y(t))]\right) d t+\int_{0}^{T}\left(p_{u}(t), D b^{n}(x) y(t)\right) d t \\
= & \int_{0}^{T}\left(p_{u}(t), y^{\prime}(t)+A y(t)-\operatorname{div} C[\varepsilon(y(t))]+D b^{n}(x) y(t)\right) d t \\
= & \int_{0}^{T}\left(p_{u}(t), B(v-u)(t)\right) d t .
\end{aligned}
$$


Let $u$ be the optimal control subject to (4.6). Then (4.10) is represented by

$$
\int_{0}^{T}\left(p_{u}(t), B(v-u)(t)\right) d t+\int_{0}^{T}(R u(t),(v-u)(t)) d t \geq 0
$$

which is rewritten by (4.8). Note that $\mathcal{C}^{*} \in B(X *, H)$ and for $\phi$ and $\psi$ in $X$ we have $\left(\mathcal{C}^{*} \Lambda_{X} \mathcal{C} \psi, \phi\right)=(\mathcal{C} \psi, \mathcal{C} \phi)$.

Remark 5.3. Identifying the antidual $M$ with $M$ we need not use the canonical isomorphism $\Lambda_{M}$. But in case where $M \subset V^{*}$ this leads to difficulties since $H$ has already been identified with its dual.

\section{References}

[1] N. U. Ahmed, Optimization and Identification of Systems Governed by Evolution Equations on Banach Space, Longman Scientific and Techniacal, 1988.

[2] J. P. Aubin, Un thèoréme de compasité, C. R. Acad. Sci. 256 (1963), 5042-5044.

[3] V. Barbu, Nonlinear Semigroups and Differential Equations in Banach Space, Nordhoff Leiden, Netherlands, 1976.

[4] - Optimal control of variational inequalities, RResearch Notes in Mathematics, 100. Pitman (Advanced Publishing Program), Boston, MA, 1984.

[5] _ Analysis and Control of Nonlinear Infinite Dimensional Systems, Academic Press Limited, 1993.

[6] F. Bonnans and D. Tiba, Pontryagin's principle in the control of semilinear elliptic variational inequalities, Appl. Math. Optim. 23 (1991), no. 3, 299-312.

[7] H. Brézis, Opérateurs Maximaux Monotones et Semigroupes de Contractions dans un Espace de Hilbert, North Holland, 1973.

[8] R. Dautray and J. L. Lions, Mathematical analysis and numerical methods for science and technology, in: Evolution Problems, Vol. 5. Springer-Verlag, 1992.

[9] J. Hwang, Parameter identification problems for an extensible beam equation, J. Math. Anal. Appl. 359 (2009), no. 2, 682-695.

[10] J. Hwang and S. Nakagiri, Optimal control problems for Kirchhoff type equation with a damping term, Nonlinear Anal. 72 (2010), no. 3-4, 1621-1631.

[11] I. Karatzas and G. Stampaccia, An Introduction to Variational Inequalities and Their Applications, New York, Academic Press, 1980.

[12] J. L. Lions, Optimal Control of Systems Governed by Partial Differential Equations, Springer-Verlag Berlin new-York, 1971.

[13] J. L. Lions and E. Magenes, Non-Homogeneous Boundary Value Problems and Applications. I, II, Springer-Verlag, Berlin, Heidelberg, New york, 1971.

[14] M. Miettinen, A parabolic hemivariational inequality, Nonlinear Anal. 26 (1996), no. 4, $725-734$.

[15] M. Miettinen and P. D. Panagiotopoulos, On parabolic hemivariational inequalities and applications, Nonlinear Anal. 35 (1999), no. 7, 885-915.

[16] S. Migórski and A. Ochal, Vanishing viscosity for hemivariational inequalities modeling dynamic problems in elasticity, Nonlinear Anal. 66 (2007), no. 8, 1840-1852.

[17] J. Rauch, Discontinuous semilinear differential equations and multiple valued maps, Proc. Amer. Math. Soc. 64 (1977), no. 2, 277-282.

[18] M. Shillor, Recent Advances in Contact Mechanics, Pergamon Press, 1998.

[19] P. D. Panagiotopoulos, Inequality Problems in Mechanics and Applicatons, Convex and Nonconvex Energy Functions, Birkhäuser, Basel, Boston, 1985.

[20] - Modelling of nonconvex nonsmooth energy problems. Dynamic hemivariational inequalities with impact effects, J. Comput. Appl. Math. 63 (1995), no. 1-3, 123-138. 
[21] D. G. Park, J. M. Jeong, and S. H. Park, Regularity of parabolic hemivariational inequalities with boundary conditions, J. Inequal. \& Appl. 2009 (2009), Article ID 207873, 22 pp.

[22] J. Y. Park, H. M. Kim, and S. H. Park, On weak solutions for hyperbolic differential inclusion with discontinuous nonlinearities, Nonlinear Anal. 55 (2003), no. 1-2, 103-113.

[23] J. Y. Park and S. H. Park, On solutions for a hyperbolic system with differential inclusion and memory source term on the boundary, Nonlinear Anal. 57 (2004), no. 3, 459-472.

Jin-Mun JEONG

Department of Applied Mathematics

Pukyong National University

Busan 608-737, KorEa

E-mail address: jmjeong@pknu.ac.kr

EUN-YOUNG Ju

Department of Mathematics

Pusan NATional University

Busan 609-735, Korea

E-mail address: nabifly@hanmail.net

Hyun-Min KIM

Department of Mathematics

Pusan National University

Busan 609-735, KoreA

E-mail address: hyunmin@pusan.ac.kr 